\title{
Building a robust athlete in the South African high school sports system
}

\author{
W Lombard, MPhil, BSpSc (Hons), CSCS \& RSCC \\ Independent Athletic Performance Specialist, Co-founder LA Performance Protea Crescent West Acres, Nelspruit, 1201
}

Corresponding author: W Lombard (waynelombard@yahoo.com)

Background: School sport in South Africa has become more competitive, and competition schedules are often found to be more congested. As a consequence young athletes are in an environment where they are exposed to high training and match demands. However, the school system generally fails to prepare these athletes physically to withstand the training and competition demands placed on them.

Discussion: It is important that schools implement a system that will allow their athletes to develop physically through ageappropriate strength and conditioning. It is especially important for schools to develop a plan to manage the multi-sport athlete in order reduce the risk of injury and burnout. By adopting a five year accumulative development model that fits the athlete's environment, schools will contribute to the development of future professional athletes.

Key words: high school, student-athlete, multi-sport

\section{S Afr J Sports Med 2018; 30:1-3.DOI: 10.17159/2078-516X/2018/v30i1a2933}

Schools are presently faced with important decisions on how best to structure their sports programmes to include the growing number of tournaments into their sports calendars. Furthermore, school sports have become more professional and competitive, with the inclusion of the offer of cash prizes, as well as live TV coverage of these tournaments. Thus the pressure on coaches to win at this level has increased significantly over the past 10 years.

The intention of this article is not to disparage the South African school sport system but rather to create an understanding of best practice techniques when considering the conditioning practices related to high school athletes. As it has been the author's experience through working with numerous high school athletes and presenting to various schools and educators on this topic, that the current system or lack thereof may not be athlete-centred. This may be in turn detrimental to their sporting development at a later stage ${ }^{[1]}$.

Thus in this author's opinion, there is a definite need for a paradigm shift in school sports programming in South Africa. As it currently stands, training is generally designed for short-term competitive performance rather than focusing on the optimal long-term development of a young athlete's overall athletic capabilities ${ }^{[2]}$. With the growing volume of scientific literature in this area, it is important that this be made accessible to coaches and educators within the school system. Thus the intention of this commentary is to disseminate the science of developing school sports and presenting it simply yet meaningful with a clear emphasis on its overall purpose.

\section{Discussion}

Starting with the end in mind

It is evident that since the exclusion of physical education from the South African school curriculum, many young athletes entering high school lack the basic fine and gross motor skills that should be the norm at this age. Therefore they often start high school with a low level of physical fitness and technical ability in sports [3]. Therefore there is a need for a structured programme to address these issues and a new model of physical development is required which builds young robust athletes $[2,4]$,

Too often, young athletes are judged early on their performances during their school careers, rather than by their achievements after school. This approach may place undue pressure on the athlete to perform at a young age rather than putting in place the necessary characteristics required to sustain a high level of performance later on in their careers. Schools may need to consider the fact that as a result of different rates of maturation schoolchildren need to be developed based on their future potential and not their current sporting performance [5]. The primary aim for a school's sporting system should be to produce children who, when they are fully mature, will have the opportunity to pursue athletic achievement or lifelong recreational activity. However, the author is not implying that winning a tournament or match does not create a healthy competitive environment, but when winning is the focal point of a programme, it may be detrimental to the athlete's overall sporting development. The increase of performance pressures in school sports has resulted in a well-documented growing trend of early specialisation and overtraining of the youth ${ }^{[6]}$. School athletes are often under pressure to complete multiple sessions per day in one sport or they are multi-sport athletes who are expected to complete a full practice and match load for two sports within the same season. It is here that coaches need to look after the best interests of the athlete in terms of training load (sport specific as well as strength and conditioning) and recovery.

Consequently, an emphasis should be placed on ensuring that the youth are exposed to various age appropriate structured technical activities (all sport specific and strength training related technical abilities). This should also include tactical (an understanding of the specific tactics within a sporting code) and physical training modalities as the exposure to a wide variety of stimuli at a young age allows for greater physiological adaptations as they progress through their careers. These will help to prepare them for the demands of their sporting activities and make them more robust and resilient to injuries ${ }^{[7]}$. However, in order to do this requires an acceptance from all stakeholders (teachers, coaches, parents, trainer as well as students) and careful monitoring of the athletes' training loads and perception of wellness.

Creating an environment conducive to building a robust athlete

One of the main benefits of any high school system is that all the students are under their guidance for a minimum of five years. This allows for the development of a sound five year 
model that will allow schools to create a student-centred

approach when it comes to their sporting programmes.

The multi-sport athlete When designing a longterm plan, it is the multisport athlete (playing two or more sports) that needs careful consideration. These athletes are generally found in the middle of a battle between coaches for each sport and are let down by an ego-driven system that lacks sufficient planning and

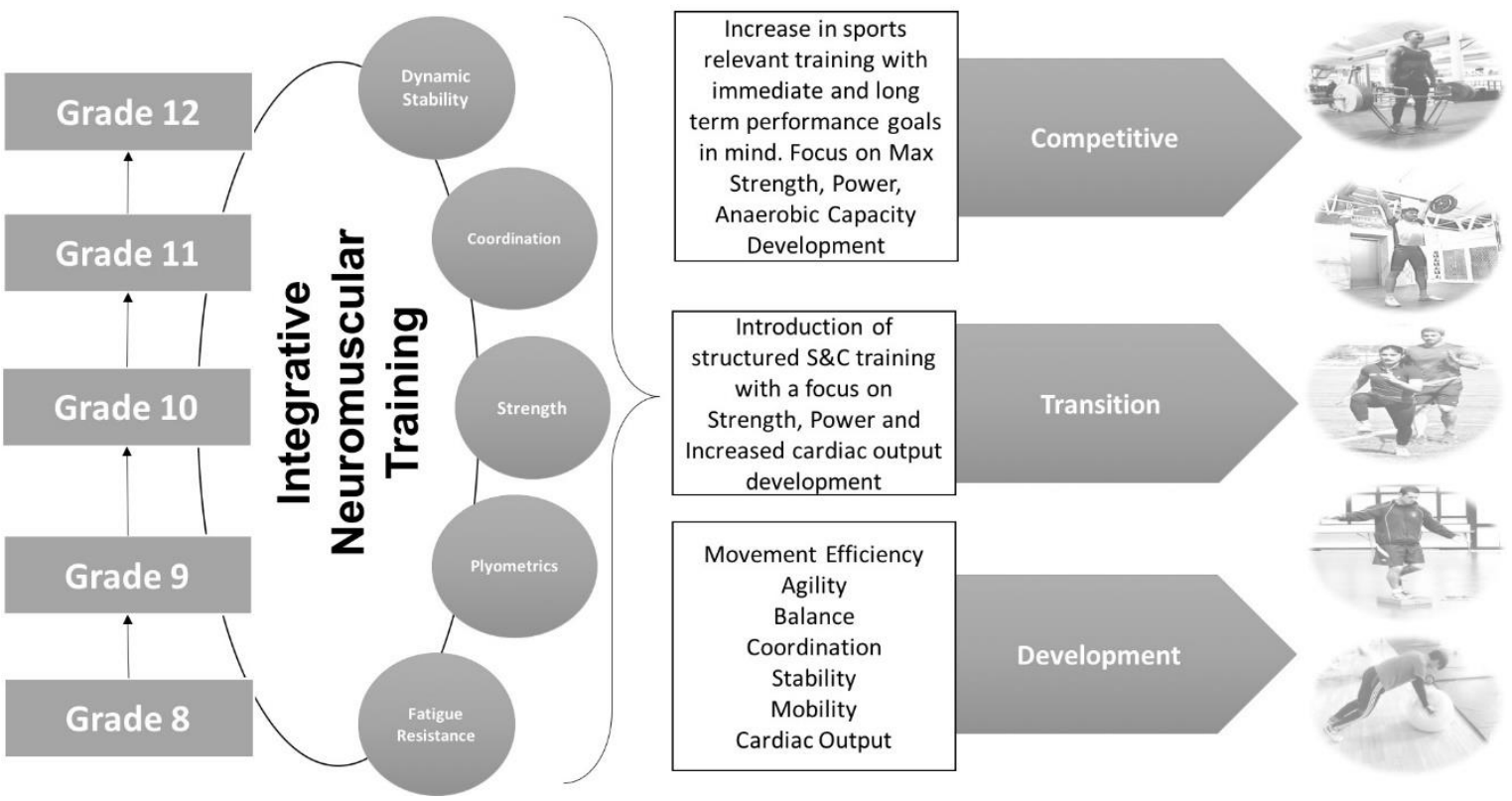
collaboration between sporting codes. It is these athletes that are often taken advantage of by coaches and are caught in a dilemma on how to prioritise each sport. It is for this reason that a well thought-out, collaborative athlete-centred periodisation plan needs to be created. The plan should be designed so that it does not favour one particular sport but rather the development of the technical skills required by each sport and the fundamental physical qualities required to be a robust athlete ${ }^{[10]}$.

\section{A five year model}

Figure 1 shows a schematic of a five year model that schools can implement to aid in the development of a robust high school athlete. Within the model there are three distinct phases that should be emphasised, (1) Development Phase (Grades 8 9), (2) Transition Phase (Grade 10) and (3) Competitive Phase (Grade 11 -12), with each phase leading into the other, thereby allowing for progressive overload and increasing complexity of training at each phase. Although beyond the scope of this commentary, it is imperative that anyone who is drawing up such a plan, takes careful consideration of what exercises are implemented at each stage of the model ${ }^{[8]}$. They should stick to the basic principles of overload, progression and specific adaptions to imposed demands (SAIDs) (please define this before using the acronym) of effective athletic development.

Fort-Vanmeerhaeghe et al. propose an integrative neuromuscular training (INT) model for youth athletes [4,7]. This model allows for the development of all fundamental physical qualities mentioned earlier for youth athletes and bodes well for training transfer into sports specific skills ${ }^{[4,7]}$. The following components should be included into the model at the three different stages mentioned earlier [4];

(1) Dynamic stability

(2) Coordination

(3) Strength

(4) Plyometrics

(5) Speed / Agility

(6) Fatigue resistant

In order for this model to work is the education of coaches, teachers and parents on the benefits of strength and conditioning for youth athletes. Many parents and coaches

hold the incorrect perception that strength training is dangerous and may stunt the athlete's growth. The scientific data show there is no evidence to support strength training stunting growth; however, it is essential that it is implemented under the guidance of a suitably qualified individual. In support of the early implementation of strength and conditioning programmes, evidence shows that the implementation of integrative neuromuscular training in youth athletes can reduce sports-related injuries to less than a third and overuse injuries by almost half [9]. Researchers have also shown that by not implementing integrative neuromuscular training during the pre-adolescence and early puberty stages of development, the probability of the athlete reaching their full genetic potential or greater is far less than those who started at a young age ${ }^{[8]}$.

\section{Conclusion}

From personal experience, it has been noticeable that most South African schools are without a long-term focused approach to developing robust high school athletes. The South African school system needs to implement an athlete-centred long-term strategy. The evidence is unequivocal, and the benefits far outweigh the negatives for the implementation of such a programme within the school system. The implementation of progressive integrative neuromuscular training from a young age will ensure that there is a reduction in the risk of injury and the production of robust athletes that are able to perform to their maximum potential with more enjoyment.

\section{References}

1. Durandt J, Hendricks S, Marshall M, et al. Under-13 rugby: What are the issues? A panel discussion. S AfrJ Sports Med 2015;27(3):63-66. [doi:10.7196/SAJSM.8078]

2. Jeffreys I. Quadrennial planning for the high school athlete. Strength Cond J 2008;30(3):74-83. [doi: 10.1519/SSC.0b013e3181775ae2]

3. Uys M, Bassett S, Draper CE, et al. Results from South Africa's 2016 report card on physical activity for children and youth. J Phys Act Health 2016;13(11 Suppl 2):S265-73. [doi: 10.1123/jpah.2016-0409]

4. Fort-Vanmeerhaeghe A, Romero-Rodriguez D, Montalvo AM, et al. Integrative neuromuscular training and injury prevention 
in youth athletes. Part I: Identifying risk factors. Strength Cond J. 2016;38(3):36-48. [doi: 10.1519/SSC.0000000000000229]

5. Lloyd RS, Oliver JL, eds. Strength and conditioning for young athletes: Science and application. New York: Routledge, pp.?

6. Jayanthi NA, LaBella CR, Fischer D, et al. Sports-specialized intensive training and the risk of injury in young athletes: a clinical case-control study. Am J Sports Med. 2015;43(4):794801. [doi: 10.1177/0363546514567298]

7. Fort-Vanmeerhaeghe A, Romero-Rodriguez D, Lloyd RS, et al. Integrative neuromuscular training in youth athletes. Part II: Strategies to prevent injuries and improve performance. Strength Cond J 2016;38(4):9-27. [doi:10.1519/SSC.0000000000000234]
8. Lloyd RS, Cronin JB, Faigenbaum AD, et al. National Strength and Conditioning Association Position Statement on LongTerm Athletic Development. J Strength Cond Res 2016;30(6):1491-1509. [doi: 10.1519/JSC.0000000000001387]

9. Abernethy L, Bleakley C. Strategies to prevent injury in adolescent sport: a systematic review. Br J Sports Med 2007;41(10):627-638. [doi: 10.1136/bjsm.2007.035691]

10. Swanson JR. Periodization for the multisport athlete. Strength Cond J 2004;26(4):50-58. 